\title{
Robotic-assisted thoracic surgery reduces perioperative complications and achieves a similar long-term survival profile as posterolateral thoracotomy in clinical N2 stage non-small cell lung cancer patients: a multicenter, randomized, controlled trial
}

\author{
Jia Huang ${ }^{1 \#}$, Yu Tian ${ }^{1 \#}$, Chongwu Li", Yaofeng Shen ${ }^{3 \#}$, Hecheng $\mathrm{Li}^{4} \wedge$, Fanzhen Lv ${ }^{5}$, Hao Lin ${ }^{1}$, Peiji Lu ${ }^{1}$, \\ Jules Lin ${ }^{6}$, Christopher Lau ${ }^{7}$, Ricardo Mingarini Terra ${ }^{8}$, Long Jiang ${ }^{1}$, Qingquan Luo ${ }^{1}$ \\ ${ }^{1}$ Shanghai Lung Cancer Center, Shanghai Chest Hospital, Shanghai Jiao Tong University, Shanghai, China; ${ }^{2}$ Department of Thoracic Surgery, \\ Shanghai Pulmonary Hospital, School of Medicine, Tongji University, Shanghai, China; ${ }^{3}$ Department of Anesthesiology, Shanghai Chest Hospital, \\ Shanghai Jiao Tong University, Shanghai, China; ${ }^{4}$ Department of Thoracic Surgery, Ruijin Hospital, Shanghai Jiao Tong University School of \\ Medicine, Shanghai, China; ${ }^{5}$ Department of Thoracic Surgery, The Affiliated Huadong Hospital of Fudan University, Shanghai, China; ${ }^{6}$ Department \\ of Surgery, Section of Thoracic Surgery, University of Michigan Medical Center, Ann Arbor, MI, USA; ${ }^{7}$ Department of Cardiothoracic Surgery, \\ Weill Cornell Medicine, New York, NY, USA; ${ }^{8}$ Thoracic Surgery Division, Heart Institute (InCor) do Hospital das Clínicas da Faculdade de \\ Medicina, Universidade de São Paulo, São Paulo, Brazil \\ Contributions: (I) Conception and design: J Huang, Q Luo, L Jiang; (II) Administrative support: Q Luo, H Li; (III) Provision of study materials \\ or patients: H Li, P Lu; (IV) Collection and assembly of data: J Huang, L Jiang, Y Shen; (V) Data analysis and interpretation: Y Tian, C Li; (VI) \\ Manuscript writing: All authors; (VII) Final approval of manuscript: All authors. \\ \#These authors contributed equally to this work. \\ Correspondence to: Qingquan Luo; Long Jiang. Shanghai Lung Cancer Center, Shanghai Chest Hospital, Shanghai Jiao Tong University, Shanghai \\ 200030, China. Email: luoqingquan@hotmail.com; dylan919@139.com.
}

Background: Our previous study demonstrated the safety and short-term efficacy of robotic-assisted thoracic surgery (RATS) in clinical N2 (c-N2) stage non-small cell lung cancer (NSCLC) patients. From this, the present study was devised, in which the follow-up time and sample size were both extended to explore the long-term efficacy and potential benefit in survival of RATS compared with lobectomy in c-N2 stage NSCLC patients.

Methods: Patients with c-N2 NSCLS were randomly assigned in a 1:1 ratio to accept operation through thoracotomy or RATS. The da Vinci Surgical System (Si/Xi) was applied in the RATS group, while conventional lobectomy with a rib-spreading incision was applied in the posterolateral thoracotomy group. Primary endpoint was defined as disease free survival and overall survival (OS) of all recruited patients.

Results: Compared with posterolateral thoracotomy group $(\mathrm{N}=72)$, the RATS group $(\mathrm{N}=76)$ had a reduced blood loss $(\mathrm{P}<0.001)$, decreased drainage duration $(\mathrm{P}=0.002)$, and decreased postoperative pain visual analog score (all $\mathrm{P}<0.001)$, but increased overall cost $(\mathrm{P}<0.001)$. Meanwhile, no difference in the other postoperative complications (such as air leakage, subcutaneous emphysema, atrial fibrillation etc.) was found between the RATS group and the posterolateral thoracotomy group (all $\mathrm{P}>0.05$ ). Regarding long-term outcome, no difference in disease-free survival (DFS; $\mathrm{P}=0.925)$ or OS $(\mathrm{P}=0.853)$ was observed between the RATS group and posterolateral thoracotomy group. Subgroup analyses and multivariable Cox regression analyses also found no difference in DFS or OS between the RATS group and posterolateral thoracotomy groups.

Conclusions: RATS reduced intraoperative bleeding, drainage duration, postoperative pain, and achieved similar long-term survival outcomes compared with posterolateral thoracotomy in c-N2 stage NSCLC patients.

^ ORCID: Yu Tian, 0000-0002-5252-9374; Hecheng Li, 0000-0001-8069-6033. 
Trial registration: Chinese Clinical Trial Registry ChiCTR-INR-17012777.

Keywords: Robotic-assisted thoracic surgery (RATS); thoracotomy; non-small cell lung cancer (NSCLC); lymph node metastasis; postoperative complications

Submitted Aug 06, 2021. Accepted for publication Nov 24, 2021.

doi: $10.21037 /$ tlcr-21-898

View this article at: https://dx.doi.org/10.21037/tlcr-21-898

\section{Introduction}

Lung cancer is the leading cause of cancer-related death worldwide, among which non-small cell lung cancer (NSCLC) accounts for nearly $80-85 \%$ of total lung cancer cases with poor prognosis, as most cases are in intermediateto-late stage when diagnosed $(1,2)$. In the management of NSCLC, surgical resection [mainly through videoassisted thoracoscopic surgery (VATS)] has become the optimal option for early-stage NSCLC patients, while for locally advanced NSCLC, multidisciplinary team (MDT) treatment, including surgery, is recommended, with thoracotomy still being the standard surgical approach (3). However, thoracotomy increases the risk of pain and postoperative infection, and is even associated with a higher rate of reoperation and surgery-related death (3). Therefore, defining the optimal surgical approach for locally advanced NSCLC is essential for improving patients' postoperative outcomes and achieving a better prognosis.

Robotic-assisted thoracic surgery (RATS), an innovative minimally invasive surgery system, provides a magnified, $3 \mathrm{D}$, high-definition surgical field for operating surgeons, allowing them to perform complicated surgery without dissecting unnecessary surrounding tissue $(4,5)$. Moreover, the maneuverability of the mechanical wrist, which is superior to that of human hand, makes radical lymphadenectomy easier $(4,5)$. Therefore, RATS may be especially suitable for patients with nodal involved disease. Previous studies indicated that RATS reduced postoperative pain compared to thoracotomy in early-stage NSCLC patients (6). Also, several retrospective studies demonstrated that RATS was feasible for stage IIIAN2 NSCLC, and an increase of robotic approach for stage IIIA-N2 disease from 3\% in 2010 to $14 \%$ in 2016, according to analyses of the National Cancer Database (NCDB) data set, has been reported (7-9). However, to the best of our knowledge, no prospective clinical trials have been conducted to assess whether RATS is comparable to thoracotomy for cancer control in locally advanced NSCLC. The short-term outcomes of the comparison have been reported previously, showing that RATS reduced blood loss, postoperative pain, and chest tube duration compared to thoracotomy (10). In this article, we present the long-term outcome of our prospective clinical trial after extending the follow-up period and recruiting a larger number of patients. The current research is the first multicenter, randomized, controlled trial demonstrating the safety and efficacy of RATS in locally advanced lung cancer. The results demonstrated the comparable perioperative outcomes between RATS and conventional thoracotomy, which indicated that RATS could be considered as a surgical approach for locally advanced disease. The research also aimed to inspire that, with the advent of the era of immunotherapy, RATS could be a promising surgical method following the neoadjuvant therapy regimen. We present the following article in accordance with the CONSORT reporting checklist (available at https:// dx.doi.org/10.21037/tlcr-21-898).

\section{Methods}

\section{Study design}

This multicenter, open-label, parallel-arm, noninferiority RCT was conducted in 3 medical centers in China, including Shanghai Chest Hospital, Shanghai Ruijin Hospital, and Shanghai Huadong Hospital. The enrollment of patients was carried out between January 2016 and July 2020, and the eligible patients were randomly assigned to undergo RATS or posterolateral thoracotomy. This study was approved by the Institutional Review Board of Shanghai Chest Hospital (No. KS1735) and registered at the Chinese Clinical Trial Registry (No. ChiCTR-INR-17012777). All participants signed informed consent for publication of this manuscript and any accompanying images, in accordance with the Guidelines from the International Conference on Harmonization (11). All procedures performed in this study involving human participants were in accordance with the 
Declaration of Helsinki (as revised in 2013).

In the current study, the primary outcomes were diseasefree survival (DFS) and overall survival (OS). The secondary outcomes included operative duration, blood loss volume, drainage duration, total drainage volume, length of stay (LOS), overall cost, pain visual analogue scale (VAS) score in postoperative days (POD) 1 to 5 , and postoperative complications.

\section{Patient enrollment}

Patients were included if they were diagnosed with primary NSCLC with clinical N2 (c-N2) disease-stage according to the eighth edition of the American Joint Committee on Cancer Tumor-Node-Metastasis (TNM) classification, were 18 to 75 years old, had adequate pulmonary and cardiac function to tolerate pulmonary resection, volunteered to participate in this study, and were able give written informed consent. Positron emission tomography computed tomography (PET-CT) and biopsy through endobronchial ultrasound guided transbronchial needle aspiration (EBUS-TBNA) or mediastinoscopy were recommended if the patients were willing. All the tumors of included patients were evaluated as resectable by the MDT. If the above examinations were absent, patients with enlarged mediastinal lymph nodes (diameter more than 1 $\mathrm{cm}$ ) on computed tomography could be included after they were assessed by MDT.

The intraoperative exclusion criteria were as follows: (I) pathological results other than NSCLC through intraoperative frozen section examination; (II) pleural dissemination or other unexpected metastasis observed during operation; (III) change of resected range based on intraoperative exploration or unexpected event.

\section{Randomization}

After patients gave written informed consent, they were registered by the local investigator at each participating institution to ensure patients' allocation and concealment. Randomization was conducted with a computer-generated random numbers table. A central randomization system was used to conduct randomization, and the eligible subjects were randomly and equally assigned to a RATS group or a posterolateral thoracotomy group in each participating center. Allocation ratio was $1: 1$. Seventy-six patients were allocated to the RATS group while 72 patients were allocated to the posterolateral thoracotomy group (Figure 1).

\section{Surgical procedure and postoperative management}

The detailed surgical procedures have been described in our previous study (10). Briefly, a radical lobectomy combined with mediastinal lymph node dissection was performed for all patients. In the RATS group, the surgery was conducted using the da Vinci Surgical System (Intuitive Surgical, Sunnyvale, CA, USA). In the posterolateral thoracotomy group, patients underwent conventional lobectomy with a rib-spreading thoracotomy of about $15 \mathrm{~cm}$. In the present study, the hilar and mediastinal lymph nodes were routinely dissected, and a minimum of 3 mediastinal lymph node stations were ultimately harvested.

An enhanced recovery after surgery (ERAS) protocol was routinely applied to both groups, including preoperative smoking cessation, breathing training, specific postoperative pain regimen, early postoperative activities and early extubation. Rehabilitation therapists were involved throughout the process of postoperative recovery for each patient. Patient-controlled analgesia and nonsteroidal antiinflammatory drugs were applied routinely for patients after surgery. The intravenous nonsteroidal anti-inflammatory drugs, meperidine and tramadol, were occasionally used when necessary. The ERAS protocol was identical in both groups.

\section{Data collection}

Before the operation, basic clinical characteristics of patients were recorded, including age, gender, history of smoking, comorbidities, forced expiratory volume in the first second $\left(\mathrm{FEV}_{1} ; \%\right)$, diffusing capacity for carbon monoxide (DLCO; \%), gene mutation status of epidermal growth factor receptor $(E G F R)$, and tumor location. After surgery, histologic examination was routinely performed to assess histologic type, tumor size, and pathologic TNM stage. Meanwhile, operation-related characteristics were also documented, which included operative duration, blood loss volume, drainage duration, total drainage volume, LOS, and overall cost. In addition, visual analogue pain scores were recorded from POD 1 to 5. Postoperative complications were also documented in detail.

\section{Follow-up}

After surgery, patients were reviewed in the outpatient clinic every 3 months for the first 2 years, and every 6 months thereafter. DFS and OS were estimated: DFS was defined as the duration from surgery to disease relapse or patient's 


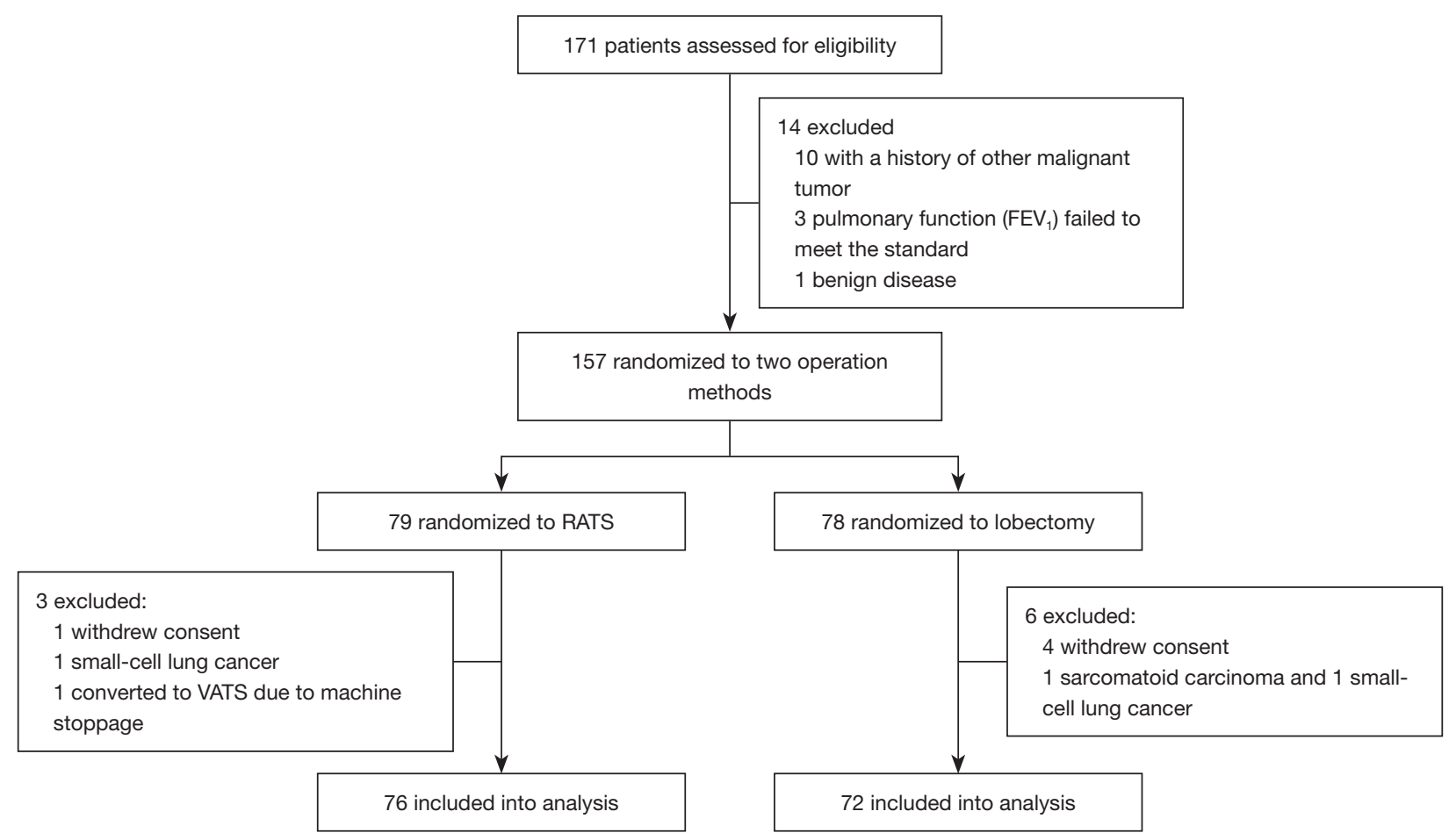

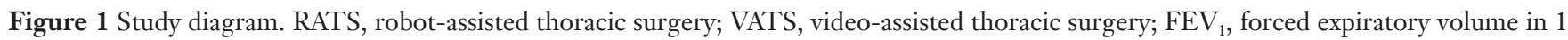
second.

death; OS was defined as the duration from surgery to patient's death. Patients who were lost to follow-up were censored on the date they were last contacted.

\section{Statistical analysis}

Based on our clinical observations and review of the previous research, a sample size was determined according to the 3 -year OS after RATS and thoracotomy, and 80\% power with a $5 \%$ noninferiority margin and a significance level of $5 \%$ was ensured. Statistical analysis was carried out using SPSS 22.0 (IBM Corporation, Armonk, NY, USA). Graph plotting was completed using GraphPad Prism 7.02 (GraphPad Software Inc., San Diego, CA, USA). Data are described as mean values with standard deviation (SD), median with interquartile range (IQR), or numbers with percentage. Analyses between 2 groups was conducted with Student's $t$ test, Wilcoxon rank-sum test, chi-square test, and Fisher's exact test, as appropriate. A survival curve was plotted using the Kaplan-Meier method and analyzed by log-rank (MantelCox) test. Factors related to DFS and OS were analyzed by Cox's regression model analyses. A 2-sided $\mathrm{P}$ value less than 0.05 was considered to be statistically significant.

\section{Results}

\section{Baseline characteristics}

Between January 2016 and July 2020, 148 patients were enrolled for randomization. The mean ages of the RATS group and posterolateral thoracotomy group were $60.9 \pm 9.4$ and $61.0 \pm 7.6$ years, respectively (Table 1 ). There were $51(67.1 \%)$ males and $25(32.9 \%)$ females in the RATS group, and $51(70.8 \%)$ males and $21(29.2 \%)$ females in posterolateral thoracotomy group. Regarding the number of positive mediastinal LN stations, there were 51 $(67.1 \%)$ and $43(59.7 \%)$ cases with negative mediastinal LN stations, 7 (9.2\%) and $9(12.5 \%)$ cases with a single positive mediastinal LN station, and 18 (23.7\%) and 20 (27.8\%) with multiple positive mediastinal LN stations in the RATS group and posterolateral thoracotomy group, respectively. By comparison, no difference of age $(\mathrm{P}=0.911)$, gender $(\mathrm{P}=0.624)$, history of smoking $(\mathrm{P}=0.194)$, hypertension $(\mathrm{P}=0.487)$, diabetes $(\mathrm{P}=0.162)$, cardiovascular and cerebrovascular disease $(\mathrm{P}=0.684)$, chronic obstructive pulmonary disease $(\mathrm{P}=1.000)$, complications $(\mathrm{P}=0.951)$, $\mathrm{FEV}_{1}(\mathrm{P}=0.716)$, DLCO $(\mathrm{P}=0.119)$, occurrence of $E G F R$ gene mutation $(\mathrm{P}=0.267)$, tumor location $(\mathrm{P}=0.572)$, 
Table 1 Clinicopathological characteristics of patients

\begin{tabular}{|c|c|c|c|}
\hline Characteristics & RATS (N=76) & Posterolateral thoracotomy $(\mathrm{N}=72)$ & $P$ value \\
\hline Gender, n (\%) & & & 0.624 \\
\hline Male & $51(67.1)$ & $51(70.8)$ & \\
\hline Female & $25(32.9)$ & $21(29.2)$ & \\
\hline \multicolumn{4}{|l|}{ Complications, n (\%) } \\
\hline Hypertension & $24(31.6)$ & $19(26.4)$ & 0.487 \\
\hline Diabetes & $3(3.9)$ & $7(9.7)$ & 0.162 \\
\hline CCVD & $5(6.6)$ & $6(8.3)$ & 0.684 \\
\hline $\mathrm{FEV}_{1}(\%)$, mean $\pm \mathrm{SD}$ & $89.0 \pm 14.1$ & $90.0 \pm 16.2$ & 0.716 \\
\hline DLCO $(\%)$, mean \pm SD & $94.1 \pm 16.5$ & $89.6 \pm 15.8$ & 0.119 \\
\hline Gene mutation of EGFR, n (\%) & $23(30.3)$ & $16(22.2)$ & 0.267 \\
\hline Tumor location, n (\%) & & & 0.572 \\
\hline Left & $18(23.7)$ & $18(25.0)$ & \\
\hline Middle & 0 & $1(1.4)$ & \\
\hline Right & $58(76.3)$ & $53(73.6)$ & \\
\hline Histologic subtype, n (\%) & & & 0.159 \\
\hline Tumor size $(\mathrm{cm})$, mean \pm SD & $3.3 \pm 1.4$ & $3.6 \pm 1.5$ & 0.242 \\
\hline Number of positive mediastinal LN stations, $\mathrm{n}(\%)$ & & & 0.629 \\
\hline 0 & $51(67.1)$ & $43(59.7)$ & \\
\hline 1 & $7(9.2)$ & $9(12.5)$ & \\
\hline$\geq 2$ & $18(23.7)$ & $20(27.8)$ & \\
\hline Pathological TNM stage, n (\%) & & & 0.342 \\
\hline I & $24(31.6)$ & $21(29.2)$ & \\
\hline II & $24(31.6)$ & $17(23.6)$ & \\
\hline III & $27(35.5)$ & $33(45.8)$ & \\
\hline IV & $1(1.3)$ & $1(1.4)$ & \\
\hline
\end{tabular}

Table 1 (continued) 
Table 1 (continued)

\begin{tabular}{|c|c|c|c|}
\hline Characteristics & RATS (N=76) & Posterolateral thoracotomy $(\mathrm{N}=72)$ & $P$ value \\
\hline \multicolumn{4}{|l|}{ Adjuvant therapy, n (\%) } \\
\hline Chemotherapy & $26(34.2)$ & $21(29.2)$ & 0.510 \\
\hline Chemoradiotherapy & $4(5.3)$ & $6(8.3)$ & 0.457 \\
\hline Others $^{c}$ & $3(3.9)$ & $2(2.8)$ & 1.000 \\
\hline
\end{tabular}

others $^{\mathrm{a}}$, bronchiectasis, gout, fatty liver disease, hepatitis B, and bladder cancer; others ${ }^{\mathrm{b}}$, typical carcinoid, lymphoepithelioma-like carcinoma, and pleomorphic carcinoma; others ${ }^{c}$, immunotherapy and target therapy. RATS, robotic-assisted thoracic surgery; SD, standard deviation; CCVD, cardiovascular and cerebrovascular disease; COPD, chronic obstructive pulmonary disease; FEV ${ }_{1}$, forced expiratory volume in $1 \mathrm{~s}$; DLCO, carbon monoxide diffusion capacity; EGFR, epidermal growth factor receptor; ADC, adenocarcinoma; SCC, squamous cell carcinoma; ASC, adenosquamous carcinoma; LN, lymph nodes.

Table 2 Operation-related characteristics

\begin{tabular}{|c|c|c|c|}
\hline Characteristics & RATS (N=76) & Posterolateral thoracotomy $(\mathrm{N}=72)$ & $P$ value \\
\hline Blood loss, n (\%) & & & $<0.001$ \\
\hline$<100 \mathrm{~mL}$ & $65(85.5)$ & $16(22.2)$ & \\
\hline$\geq 100 \mathrm{~mL}$ & $11(14.5)$ & $56(77.8)$ & \\
\hline Total drainage volume $(\mathrm{mL})$, median (IQR) & $855.0(602.5-1,167.5)$ & $920.0(592.5-1,646.3)$ & 0.146 \\
\hline LOS (days), median (IQR) & $10.0(8.0-13.0)$ & $11.0(9.0-14.8)$ & 0.054 \\
\hline Overall cost ( $¥)$, median (IQR) & $100,453.7(88,669.5-111,794.9)$ & $77,235.4(67,053.7-90,431.2)$ & $<0.001$ \\
\hline
\end{tabular}

RATS, robotic-assisted thoracic surgery; SD, standard deviation; IQR, interquartile range; LOS, length of hospital stay

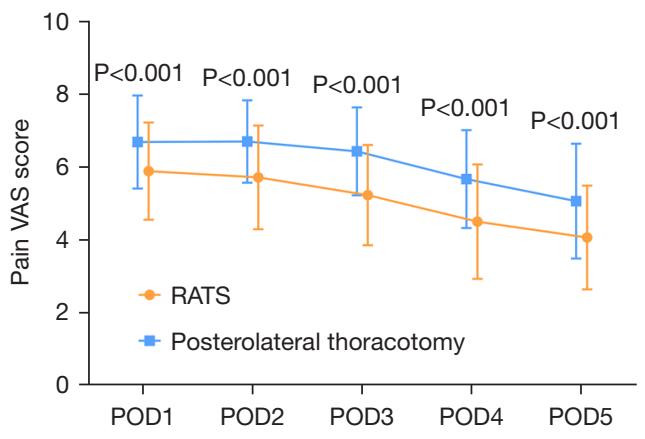

Figure 2 RATS reduced postoperative VAS score compared to posterolateral thoracotomy. VAS, visual analogue scale; RATS, robot-assisted thoracic surgery; POD, postoperative day.

histological subtype $(\mathrm{P}=0.159)$, tumor size $(\mathrm{P}=0.242)$, number of positive mediastinal $\mathrm{LN}$ stations $(\mathrm{P}=0.629)$, pathological TNM stage $(\mathrm{P}=0.342)$, chemotherapy $(\mathrm{P}=0.510)$, chemoradiotherapy $(\mathrm{P}=0.457)$, or other adjuvant therapy ( $\mathrm{P}=1.000)$ was observed between the RATS group and posterolateral thoracotomy group. The detailed clinical information is summarized in Table 1 .

\section{Perioperative outcomes}

Compared with the posterolateral thoracotomy group, the RATS group had lower blood loss $(\mathrm{P}<0.001)$ and drainage duration $(\mathrm{P}=0.002)$ but increased overall cost $(\mathrm{P}<0.001)$ (Table 2). However, there was no difference in operative duration $(\mathrm{P}=0.757)$, total drainage volume $(\mathrm{P}=0.146)$, or LOS $(\mathrm{P}=0.054)$ between the RATS group and posterolateral thoracotomy group. According to the VAS score, the RATS group had a reduced VAS score from POD1 to POD5 compared with the thoracotomy group (all $\mathrm{P}<0.001$; Figure 2).

By comparison, there was no difference in postoperative complications between the RATS group and posterolateral thoracotomy group (all $\mathrm{P}>0.05$ ). The most common 
Table 3 Postoperative complications.

\begin{tabular}{|c|c|c|c|}
\hline Complications & RATS $(\mathrm{N}=76)$ & Posterolateral thoracotomy $(\mathrm{N}=72)$ & $P$ value \\
\hline Bronchopleural fistula & $4(5.3)$ & $1(1.4)$ & 0.367 \\
\hline Pneumonia & $3(3.9)$ & $6(8.3)$ & 0.318 \\
\hline Atrial fibrillation & $3(3.9)$ & $4(5.6)$ & 0.714 \\
\hline Chest tube reinsertion & $3(3.9)$ & $4(5.6)$ & 0.714 \\
\hline Subcutaneous emphysema & $3(3.9)$ & $2(2.8)$ & 1.000 \\
\hline Chylothorax & $3(3.9)$ & $2(2.8)$ & 1.000 \\
\hline Hyperpyrexia & $2(2.6)$ & $6(8.3)$ & 0.158 \\
\hline Pulmonary embolism & $1(1.3)$ & 0 & 1.000 \\
\hline Pyothorax & 0 & $1(1.4)$ & 0.486 \\
\hline ARDS & 0 & $1(1.4)$ & 0.486 \\
\hline
\end{tabular}

Data presented as $\mathrm{n}(\%)$. RATS, robotic-assisted thoracic surgery; ARDS, acute respiratory distress syndrome.
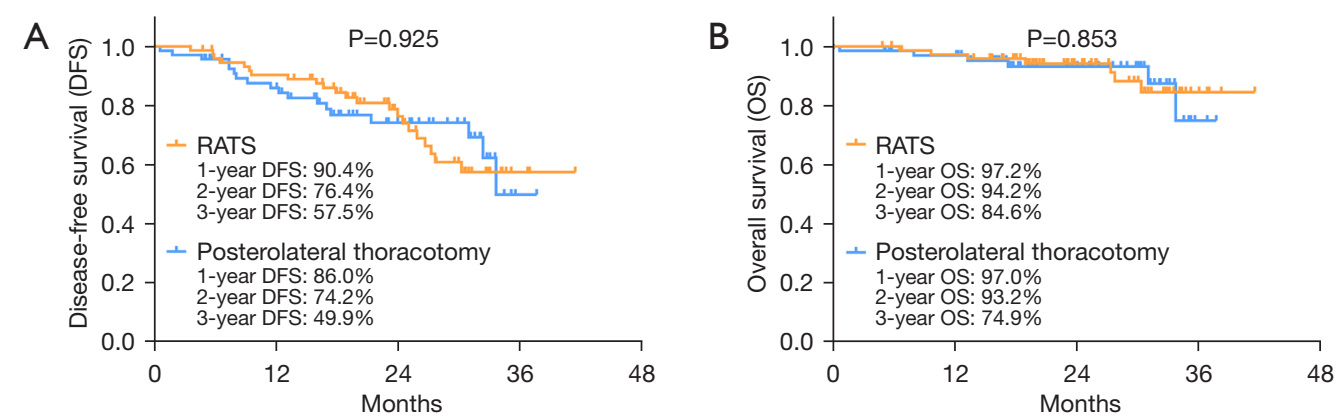

Figure 3 The RATS group showed similar survival profiles as did the posterolateral thoracotomy group. Comparison of DFS (A) and OS (B) between the RATS group and posterolateral thoracotomy group. RATS, robot-assisted thoracic surgery; DFS, disease-free survival; OS, overall survival.

postoperative complications that occurred in the RATS group were prolonged air leak $(7.9 \%)$ and bronchopleural fistula (5.3\%; Table 3). Meanwhile, in the posterolateral thoracotomy group, commonly observed postoperative complications were hyperpyrexia $(8.3 \%)$, prolonged air leak $(8.3 \%)$, pneumonia $(8.3 \%)$, atrial fibrillation $(5.6 \%)$, chest tube reinsertion (5.6\%), and recurrent laryngeal nerve injury $(5.6 \%)$, and the above-mentioned complications were mild and manageable. Overall, patients undergoing RATS had less surgical injury and better recovery during hospitalization without increasing the postoperative complications.

\section{Survival outcomes}

There was no difference in DFS $(\mathrm{P}=0.925$; Figure $3 A)$ or OS $(\mathrm{P}=0.853$; Figure $3 B)$ between the RATS and thoracotomy groups. Further subgroup analyses indicated that the survival profile did not differ between the RATS group and posterolateral thoracotomy group in terms of tumor size (Figure 4A-D) and pathological TNM stage (Figure 4E-H). Multivariable Cox regression analysis revealed that 

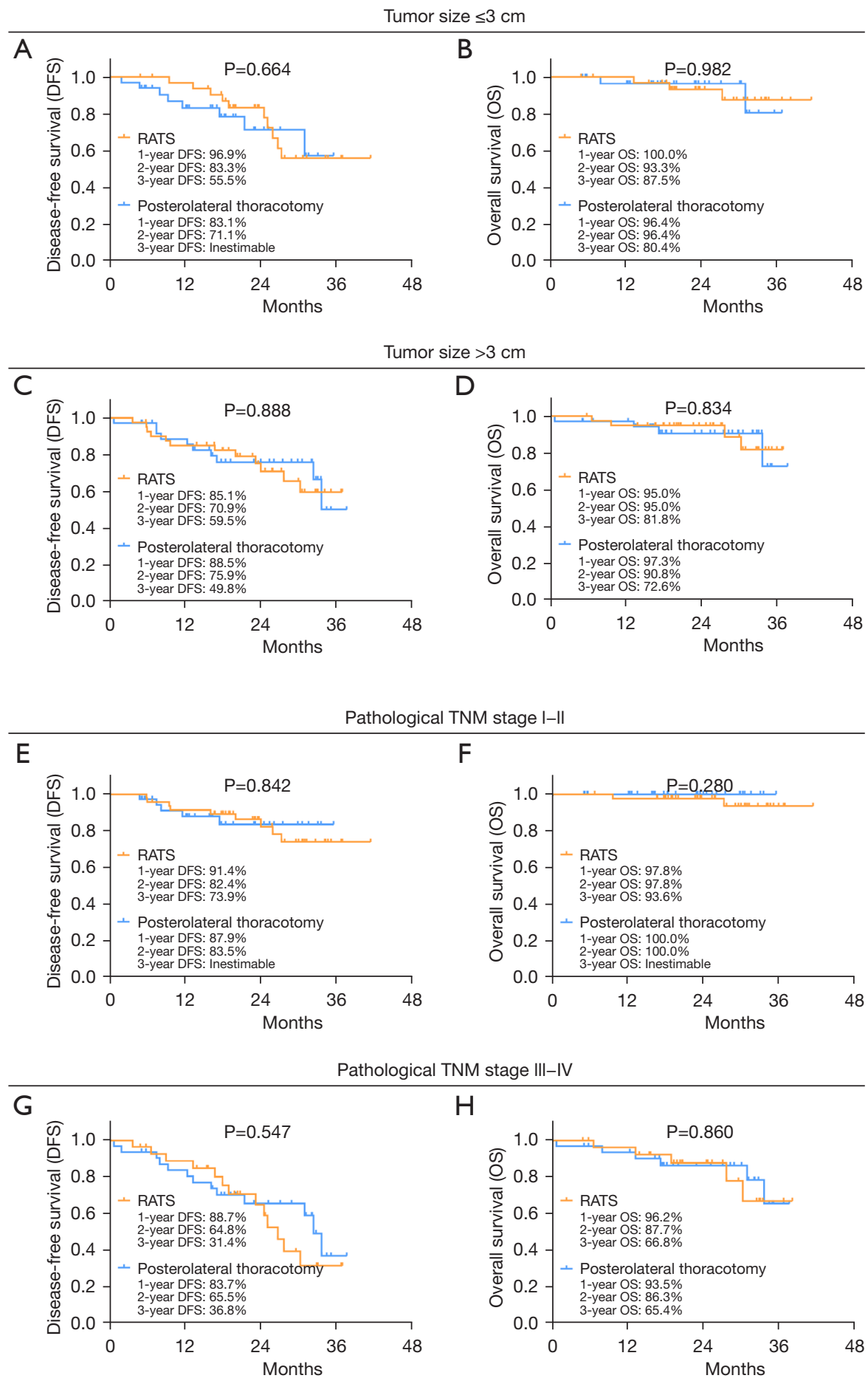

Figure 4 Subgroup analyses. Comparison of DFS (A) and OS (B) between the RATS group and posterolateral thoracotomy group in NSCLC patients with a tumor size smaller than $3 \mathrm{~cm}$. Comparison of DFS (C) and OS (D) between the RATS group and posterolateral thoracotomy group in NSCLC patients with a tumor size larger than $3 \mathrm{~cm}$. Comparison of DFS (E) and OS (F) between the RATS group and posterolateral thoracotomy group in NSCLC patients with pathological TNM stage I-II. Comparison of DFS (G) and OS (H) between the RATS group and posterolateral thoracotomy group in NSCLC patients with pathological TNM stage III-IV. NSCLC, non-small cell lung cancer; RATS, robot-assisted thoracic surgery; DFS, disease-free survival; OS, overall survival. 
Table 4 Cox's proportional hazards regression model analysis for DFS

\begin{tabular}{|c|c|c|c|c|}
\hline \multirow{2}{*}{ Parameters } & \multicolumn{4}{|c|}{ Multivariate Cox's regression analysis } \\
\hline & $P$ value & $\mathrm{HR}$ & Lower & Upper \\
\hline Surgical type (RATS vs. posterolateral thoracotomy) & 0.824 & 0.927 & 0.478 & 1.801 \\
\hline Gender (male vs. female) & 0.058 & 2.727 & 0.968 & 7.684 \\
\hline Histologic subtype (ADC vs. SCC) & 0.331 & 1.571 & 0.632 & 3.905 \\
\hline Tumor size (>3 vs. $\leq 3 \mathrm{~cm}$ ) & 0.488 & 0.781 & 0.388 & 1.572 \\
\hline Mediastinal LN (positive vs. negative) & $<0.001$ & 4.966 & 2.191 & 11.254 \\
\hline Adjuvant therapy (yes vs. no) & 0.041 & 0.448 & 0.208 & 0.967 \\
\hline
\end{tabular}

DFS, disease-free survival; HR, hazard ratio; $\mathrm{Cl}$, confidence interval; RATS, robotic-assisted thoracic surgery; ADC, adenocarcinoma; SCC, squamous cell carcinoma; LN, lymph node.

Table 5 Cox's proportional hazards regression model analysis for OS

\begin{tabular}{|c|c|c|c|c|}
\hline \multirow{2}{*}{ Parameters } & \multicolumn{4}{|c|}{ Multivariate Cox's regression analysis } \\
\hline & $P$ value & $\mathrm{HR}$ & Lower & Upper \\
\hline Surgical type (RATS vs. posterolateral thoracotomy) & 0.781 & 1.172 & 0.383 & 3.588 \\
\hline Gender (male vs. female) & 0.208 & 5.076 & 0.404 & 63.740 \\
\hline Histologic subtype (ADC vs. SCC) & 0.658 & 1.364 & 0.346 & 5.374 \\
\hline Tumor size (>3 vs. $\leq 3 \mathrm{~cm}$ ) & 0.913 & 1.072 & 0.308 & 3.730 \\
\hline Mediastinal LN (positive vs. negative) & 0.005 & 8.935 & 1.923 & 41.520 \\
\hline Adjuvant therapy (yes vs. no) & 0.149 & 0.382 & 0.103 & 1.413 \\
\hline
\end{tabular}

OS, overall survival; HR, hazards ratio; $\mathrm{Cl}$, confidence interval; RATS, robotic-assisted thoracic surgery; ADC, adenocarcinoma; SCC, squamous cell carcinoma; LN, lymph nodes.

the surgical type (RATS $v s$. posterolateral thoracotomy) was not correlated with DFS [hazard ratio (HR) 0.927; $\mathrm{P}=0.824$; Table 4). However, positive mediastinal lymph node independently correlated with reduced DFS (HR 4.966; $\mathrm{P}<0.001$ ), while adjuvant therapy independently correlated with prolonged DFS (HR 0.448; $\mathrm{P}=0.041$ ). Moreover, surgical approach (RATS vs. posterolateral thoracotomy) was not independently correlated with OS (HR 1.172, P=0.781; Table 5), while mediastinal lymph node invasion was an independent risk factor of decreased OS (HR 8.935; $\mathrm{P}=0.005)$. RATS, as a less invasive surgical approach, had a similar long-term result with lobectomy in treating the $\mathrm{N} 2$ disease.

\section{Discussion}

The application of RATS is becoming increasingly common in several surgical disciplines (such as urology, gynecology, and cardiac surgery) owing to its optimal operability and minimal invasiveness (12-15). Previous studies have indicated that RATS reduces intraoperative bleeding, chest tube drainage, and hospitalization stay compared to thoracotomy in early-stage NSCLC patients (15-17). RATS has also been shown to reduce postoperative pain compared to thoracotomy in stage I-IIIA NSCLC patients (18). Regarding safety, postoperative complications of RATS have been demonstrated to be similar those of thoracotomy 
in early-stage NSCLC patients $(17,19)$. However, RATS may increase the overall cost and incur a large economic burden on patients $(18,20,21)$. As for the oncological efficacy of RATS in locally advanced NSCLC patients, several retrospective studies suggest that RATS lobectomy is safe and effective in patients with N2 disease (7-9). VATS is also a minimally invasive surgical approach that is often compared to RATS. In the present study, VATS was not included as a control group because its oncological efficacy in untreated locally advanced NSCLC is suboptimal. By comparison, conventional thoracotomy, which has been proven to be an effective approach, was more appropriate for examining the noninferiority of RATS for c-N2 disease. To our knowledge, this study is the first prospective randomized clinical trial to compare the safety and survival outcome of RATS and thoracotomy for clinical N2 disease.

Consistent with previous retrospective studies, we found that RATS could reduce blood loss, chest tube duration, and postoperative pain while maintaining similar postoperative complication rates compared to posterolateral thoracotomy in c-N2 stage NSCLC patients. However, RATS increased the overall cost compared to posterolateral thoracotomy in c-N2 stage NSCLC patients partly due to the high price of purchase and maintenance of the robot as well as the instruments and disposables (21).

Despite great interest in the long-term outcome after lobectomy through RATS in NSCLC patients, few relevant studies have been conducted due to this procedure's shortduration of clinical application. One study reported there to be no difference in DFS or OS between NSCLC patients undergoing RATS and those undergoing thoracotomy, and further analysis suggested the surgical approach to not be correlated with long-term prognosis in clinical stage I NSCLC patients (22). Other researchers found no difference in DFS or OS between RATS and thoracotomy for clinical N0 stage NSCLC patients (23), and no difference in 5-year OS across stage I-IIIA NSCLC patient treated with RATS, VATS, or thoracotomy (6). As for locally advanced NSCLC, one study discovered that minimally invasive surgical approaches (VATS and RATS) and thoracotomy displayed comparable 3-year DFS and OS (8). Consistent with reports in early-stage NSCLC patients, we discovered that c-N2 stage NSCLC patients who underwent RATS experienced a similar survival profile as those who received thoracotomy. Furthermore, surgical type (RATS $v s$. thoracotomy) did not significantly correlate with DFS or OS in c-N2 stage NSCLC patients in the current study. This may also be explained by the fact that a large proportion of NSCLC patients had negative N2 nodal involvement after $\mathrm{LN}$ dissection and thus might have shared similar DFS and OS regardless of the surgical approach. Taken together, RATS displayed rather promising oncological effect in locally advanced NSCLC, which was comparable with that of thoracotomy.

Discovering the potential risk factors for NSCLC patients is critical for clinicians to identify patients with high recurrence risk, who could benefit from neoadjuvant therapy and early adjuvant therapy to obtain better prognosis. In the present study, we discovered that the presence of positive mediastinal lymph nodes independently correlated with lower DFS, while adjuvant therapy independently correlated with prolonged DFS in NSCLC patients.

These results suggested that lymph node metastasis may be a critical factor for poor prognosis and that NSCLC of N2 stage could be a systemic disease rather than just locally advanced malignant tumor. While radical surgery plays an important role in treatment, defining the optimal therapeutic regimen is critical to improve prognosis. In the current study, adjuvant chemotherapy was associated with prolonged DFS in NSCLC patients, which was consistent with previous studies, and may be important in eliminating any residual micro-metastatic disease after surgery (24). As research on neoadjuvant treatment progresses, novel induction therapy regimens may be a key approach to achieve a better prognosis. Neoadjuvant immunotherapy combined with chemotherapy in particular may represent a breakthrough in extending the survival of patients with $\mathrm{N} 2$ disease. Future research is expected to examine the combination of neoadjuvant therapy, which can eliminate micro-metastatic lesions, and RATS, which can provide a minimally invasive approach with radical tumor resection and adequate lymph node dissection.

There were some limitations in the present study. First, the median follow-up period in our study was 23.5 months with a range of $0.6-41.5$ months, which may not be long enough to fully reveal the difference. Since the survival results showed no difference between RATS and VATS, and thus further study with a longer follow-up period (such as 3 or 5 years) is needed to clarify any potential survival difference. Second, the sample size in the present study was relatively small; therefore, further studies with larger sample sizes are needed to validate the long-term outcome of RATS in c-N2 stage NSCLC patients. Finally, 
although invasive lymph node biopsy is recommended for c-N2 stage NSCLC patients prior to surgery to obtain accurate diagnosis and suitable management, a considerable proportion of patients were N1-stage and even $\mathrm{N}$ negative, as it is difficult to practice invasive and costly examination in the real clinical setting.

In conclusion, RATS was associated with reduced blood loss, drainage duration, and postoperative pain while conferring a similar long-term survival profile compared to posterolateral thoracotomy, thus supporting its potential value in treatment of $\mathrm{c}-\mathrm{N} 2$ stage NSCLC.

\section{Acknowledgments}

The authors appreciate the academic support from AME Robotic-assisted Thoracic Surgery (RATS) Collaborative Group.

Funding: This work was supported by Shanghai Hospital Development Center (Grant Number: SHDC12016113) and the National Natural Science Foundation of China (No. 81972176).

\section{Footnote}

Reporting Checklist: The authors have completed the CONSORT reporting checklist. Available at https://dx.doi. org/10.21037/tlcr-21-898

Trial Protocol: Available at https://dx.doi.org/10.21037/tlcr21-898

Data Sharing Statement: Available at https://dx.doi. org/10.21037/tlcr-21-898

Conflicts of Interest: All authors have completed the ICMJE uniform disclosure form (available at https://dx.doi. org/10.21037/tlcr-21-898). The authors have no conflicts of interest to declare.

Ethical Statement: The authors are accountable for all aspects of the work in ensuring that questions related to the accuracy or integrity of any part of the work are appropriately investigated and resolved. The Institutional Review Board of Shanghai Chest Hospital approved the study (No. KS1735). Written informed consent was obtained from the patient for publication of this manuscript and any accompanying images. All procedures performed in this study involving human participants were in accordance with the Declaration of Helsinki (as revised in 2013).

Open Access Statement: This is an Open Access article distributed in accordance with the Creative Commons Attribution-NonCommercial-NoDerivs 4.0 International License (CC BY-NC-ND 4.0), which permits the noncommercial replication and distribution of the article with the strict proviso that no changes or edits are made and the original work is properly cited (including links to both the formal publication through the relevant DOI and the license). See: https://creativecommons.org/licenses/by-nc-nd/4.0/.

\section{References}

1. Hong QY, Wu GM, Qian GS, et al. Prevention and management of lung cancer in China. Cancer 2015;121 Suppl 17:3080-8.

2. Arenberg D. Update on screening for lung cancer. Transl Lung Cancer Res 2019;8:S77-87.

3. Duma N, Santana-Davila R, Molina JR. Non-Small Cell Lung Cancer: Epidemiology, Screening, Diagnosis, and Treatment. Mayo Clin Proc 2019;94:1623-40.

4. Jiang Y, Su Z, Liang H, et al. Video-assisted thoracoscopy for lung cancer: who is the future of thoracic surgery? J Thorac Dis 2020;12:4427-33.

5. Zirafa CC, Romano G, Key TH, et al. The evolution of robotic thoracic surgery. Ann Cardiothorac Surg 2019;8:210-7.

6. Kneuertz PJ, D'Souza DM, Richardson M, et al. LongTerm Oncologic Outcomes After Robotic Lobectomy for Early-stage Non-Small-cell Lung Cancer Versus Videoassisted Thoracoscopic and Open Thoracotomy Approach. Clin Lung Cancer 2020;21:214-224.e2.

7. Park BJ, Yang HX, Woo KM, et al. Minimally invasive (robotic assisted thoracic surgery and video-assisted thoracic surgery) lobectomy for the treatment of locally advanced non-small cell lung cancer. J Thorac Dis 2016;8:S406-13.

8. Casiraghi M, Spaggiari L. Robotic resection of stage III lung cancer: an international retrospective study. J Thorac Dis 2018;10:S3081-3.

9. Herb JN, Kindell DG, Strassle PD, et al. Trends and Outcomes in Minimally Invasive Surgery for Locally Advanced Non-Small-Cell Lung Cancer With N2 Disease. Semin Thorac Cardiovasc Surg 2021;33:547-55.

10. Huang J, Li C, Li H, et al. Robot-assisted thoracoscopic surgery versus thoracotomy for c-N2 stage NSCLC: short-term outcomes of a randomized trial. Transl Lung 
Cancer Res 2019;8:951-8.

11. Branch SK. Guidelines from the International Conference on Harmonisation (ICH). J Pharm Biomed Anal 2005;38:798-805.

12. Lo EM, Kim HL. Robot-Assisted Surgery for Upper Tract Urothelial Carcinoma. Urol Clin North Am 2021;48:71-80.

13. Zanagnolo V, Garbi A, Achilarre MT, et al. Robotassisted Surgery in Gynecologic Cancers. J Minim Invasive Gynecol 2017;24:379-96.

14. Ishikawa N, Watanabe G. Robot-assisted cardiac surgery. Ann Thorac Cardiovasc Surg 2015;21:322-8.

15. Kanzaki M. Current status of robot-assisted thoracoscopic surgery for lung cancer. Surg Today 2019;49:795-802.

16. Veronesi G, Novellis P, Voulaz E, et al. Robot-assisted surgery for lung cancer: State of the art and perspectives. Lung Cancer 2016;101:28-34.

17. Hu J, Chen Y, Dai J, et al. Perioperative outcomes of robot-assisted vs video-assisted and traditional open thoracic surgery for lung cancer: A systematic review and network meta-analysis. Int J Med Robot 2020;16:1-14.

18. Veluswamy RR, Whittaker Brown SA, Mhango G, et al. Comparative Effectiveness of Robotic-Assisted Surgery for Resectable Lung Cancer in Older Patients. Chest 2020;157:1313-21.

Cite this article as: Huang J, Tian Y, Li C, Shen Y, Li H, Lv F, Lin H, Lu P, Lin J, Lau C, Terra RM, Jiang L, Luo Q. Robotic-assisted thoracic surgery reduces perioperative complications and achieves a similar long-term survival profile as posterolateral thoracotomy in clinical N2 stage non-small cell lung cancer patients: a multicenter, randomized, controlled trial. Transl Lung Cancer Res 2021;10(11):4281-4292. doi: 10.21037/tlcr-21-898
19. Hu X, Wang M. Efficacy and Safety of Robot-assisted Thoracic Surgery (RATS) Compare with Video-assisted Thoracoscopic Surgery (VATS) for Lung Lobectomy in Patients with Non-small Cell Lung Cancer. Comb Chem High Throughput Screen 2019;22:169-78.

20. Novellis P, Bottoni E, Voulaz E, et al. Robotic surgery, video-assisted thoracic surgery, and open surgery for early stage lung cancer: comparison of costs and outcomes at a single institute. J Thorac Dis 2018;10:790-8.

21. Novellis P, Alloisio M, Vanni E, et al. Robotic lung cancer surgery: review of experience and costs. J Vis Surg 2017;3:39.

22. Yang HX, Woo KM, Sima CS, et al. Long-term Survival Based on the Surgical Approach to Lobectomy For Clinical Stage I Nonsmall Cell Lung Cancer: Comparison of Robotic, Video-assisted Thoracic Surgery, and Thoracotomy Lobectomy. Ann Surg 2017;265:431-7.

23. Spaggiari L, Sedda G, Maisonneuve P, et al. A Brief Report on Survival After Robotic Lobectomy for Early-Stage Lung Cancer. J Thorac Oncol 2019;14:2176-80.

24. White AA, Lee DN, Mazzola E, et al. Adjuvant therapy following induction therapy and surgery improves survival in N2-positive non-small cell lung cancer. J Surg Oncol 2021;123:579-86. 